\title{
Left and Right Hand Movements EEG Signals Classification Using Wavelet Transform and Probabilistic Neural Network
}

\author{
A.B.M. Aowlad Hossain, Md. Wasiur Rahman, Manjurul Ahsan Riheen \\ Department of Electronics and Communication Engineering \\ Khulna University of Engineering \& Technology, Bangladesh
}

\begin{tabular}{l} 
Article Info \\
\hline Article history: \\
Received Oct 7, 2014 \\
Revised Dec 11, 2014 \\
Accepted Dec 26, 2014 \\
\hline Keyword: \\
Artificial neural network \\
Backpropagation algorithm \\
Discrete wavelet transform \\
Electroencephalogram \\
Feature extraction \\
Probabilistic neural network
\end{tabular}

\begin{abstract}
Electroencephalogram (EEG) signals have great importance in the area of brain-computer interface (BCI) which has diverse applications ranging from medicine to entertainment. BCI acquires brain signals, extracts informative features and generates control signals from the knowledge of these features for functioning of external devices. The objective of this work is twofold. Firstly, to extract suitable features related to hand movements and secondly, to discriminate the left and right hand movements signals finding effective classifier. This work is a continuation of our previous study where beta band was found compatible for hand movement analysis. The discrete wavelet transform (DWT) has been used to separate beta band of the EEG signal in order to extract features. The performance of a probabilistic neural network (PNN) is investigated to find better classifier of left and right hand movements EEG signals and compared with classical back propagation based neural network. The obtained results shows that PNN (99.1\%) has better classification rate than the BP (88.9\%). The results of this study are expected to be helpful in brain computer interfacing for hand movements related biorehabilitation applications.
\end{abstract}

Copyright (C) 2015 Institute of Advanced Engineering and Science. All rights reserved.

\section{Corresponding Author:}

A.B.M. Aowlad Hossain,

Department of Electronics and Communication Engineering

Khulna University of Engineering \& Technology

Khulna-9203, Bangladesh.

Email: aowlad0403@ece.kuet.ac.bd

\section{INTRODUCTION}

Over a million individuals are suffering from disability annually as a result of stroke, traumatic brain or spinal cord injuries [1]. A major portion of disable people have reported troubles with hand function [2], [3]. Failure of hand function causes severe problems in leading of life for the affected persons. Recent researches show that $\mathrm{BCI}$ is a new hope in treatmenting the disabilities. EEG signal is the most trendy resource of interpreting the brain activities in the realm of non-invasive BCI. They are well studied and there is evident that they can be used in artificial hand movements [4].

EEG is graphical representation of electrical activities of brain which is recorded using electrodes locating on the scalp. EEG have certain bands with separate frequency ranges [5]: theta waves varies in the range of $4 \mathrm{~Hz}$ to $7 \mathrm{~Hz}$ and its amplitude generally arround $20 \mu \mathrm{V}$, alpha wave varies with in the range of 8 to $13 \mathrm{~Hz}$ and about $30-50 \mu \mathrm{V}$ amplitude. For beta wave, the frequencies vary between $13 \mathrm{~Hz}$ to $30 \mathrm{~Hz}$ and usually have a low voltage between 5-30 $\mu \mathrm{V}$. Different bands carry information of different brain activities. EEG signals are extensively studied by numerous researches to classify different mental or brain activities [6]-[9]. Few studies have been proposed on hand movement classifications using support vector machine (SVM), linear discrimination analysis, adaptive Gaussian coefficients, C-SVM and combination of EEG and MEG. However, most of them are computationally complex and not so effective for real time applications [10]-[14]. In this context, selection of appropriate and compatible feature is very crucial for effective 
classification with simple classifier. In our previous study on selection of proper frequency band for hand movement analysis [15], it was observed that beta band (97.5\%) has higher mapping precision and better convergence rate than the other bands, alpha (93.2\%) and theta $(87.8 \%)$. Therefore, beta band can be most suitably used for hand movement analysis. This work is an extension in order to find effective classifier of left and right hand movement EEG signal which is initiated from the findings of our previous work.

In this study, we plan to recognize left and right hand movement EEG signals finding simple but effective classifier based on wavelet transform and neural network approaches. Applying the selected features, we try to classify left and right hand movements using neural network based methods. Different features are planned to calculate using wavelet analysis of EEG signals. Wavelet transform is a powerful tool to process biomedical signals for various applications [16]. It is a robust technique to represent the signals in time-frequency domain. The capability of time-frequency domain analysis of wavelet transform can be useful to separate different bands and consequently to extract important features. Numerous techniques have been proposed for classification of bio-events or abnormalities. Artificial neural networks have been applied in several studies for EEG analysis [17]-[19]. ANNs is executed through training algorithms with specified learning criteria to imitate the learning mechanisms of biological neurons [20]. Different types and structures of neural networks are usually used. In this work, the probabilistic neural network (PNN) and classical back propagation based neural network (BP) are applied and their performances are compared to identify the left and right hand EEG signals.

The rest of the paper is organized as follows: the next section describes the proposed methodology which describes the wavelet based decomposition of EEG bands, feature extractions and two neural networks based approaches for classification. Third section presents the description of data used in this study as well as analysis and discussion on obtained results which are followed by conclusion in fourth section.

\section{METHODOLOGY}

The purpose of this study has two main steps; firstly, to extract features from beta band of EEG signals. Then, we try to detect the left and right hand movements EEG signal finding effective classifier. We used discrete wavelet transform to select beta band in order to compute different features. These features are applied to BP and PNN in order to compare their effectiveness in hand movement classification. The block diagram of this proposed methodology is shown in Figure 1.

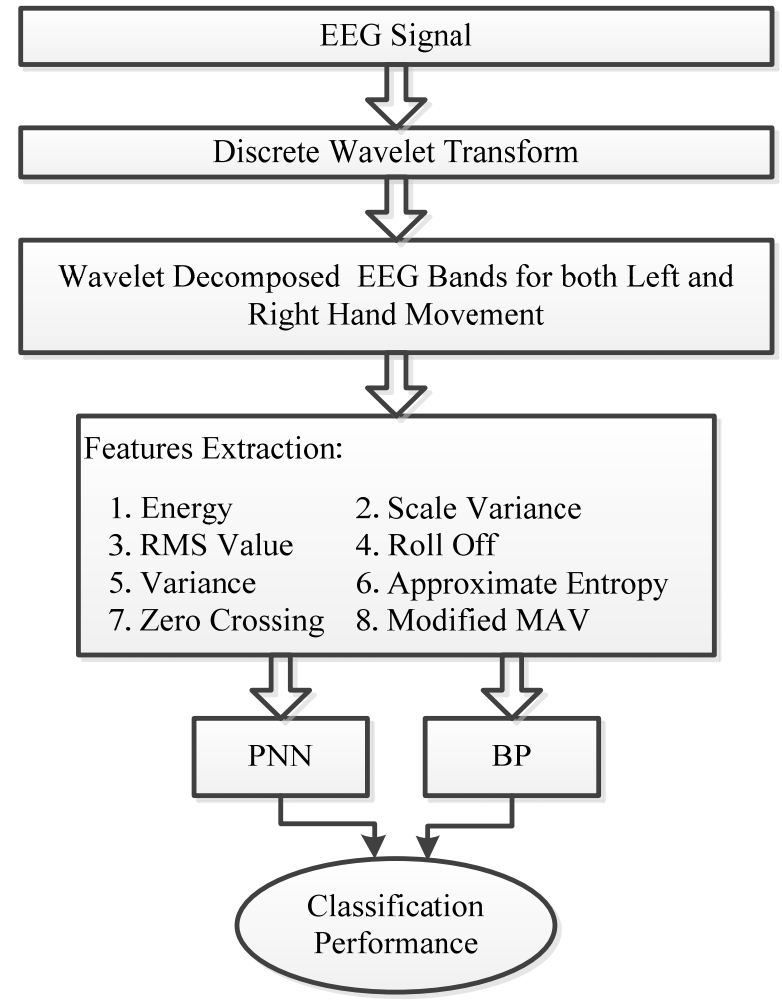

Figure 1. Block diagram for hand movements EEG signals classification 


\subsection{Wavelet Based Decomposition and Features Extraction}

The features are extracted from bio-signals can characterize the behaviors of the corresponding bioevents. Features extraction from the physiological signals is important for various applications. There are numbers of time and frequency or both domain features are generally used. A great advantage of wavelet transform based analysis is that it can represent the signal in both time and frequency domain which is better compared to the Fourier transform and short time Fourier transform. Therefore, we choose wavelet transformed signals for selecting different EEG bands signals which will be intended for features extraction.

Wavelet is a small waveform of effectively brief duration that has a zero net area and hence offering potentiality to capture events occurs in a short period of time. The wavelet transform decomposes the signal into different scales with different levels of resolution by dilating a mother wavelet. For discrete time signals, discrete wavelet transform (DWT) is equivalent to an octave filter bank [21], [22]. This multiresolution analysis (MRA) decomposes a signal into scales with different time and frequency resolution. We can discriminate the different bands of EEG signal through the decomposition of MRA into different levels. Selection of maximum decomposition levels depends on the frequency bands. Therefore, through wavelet analysis, we can conserve the time-frequency components of EEG signal at different resolution and scales. The resolution of the signal is determined by the filtering operations and the scale is determined by up sampling and down sampling operations. As a result, the DWT can be achieved by successive low pass and high pass filter at discrete time domain is shown in Fig. 2. Where $x[n]$ is the input signal, which passes through a high pass filter of impulse response $h[n]$ and simultaneously passes through the low pass filter with an impulse response $g[n]$. The output of the high pass filter provides the detail coefficients $D$ and the output of the low pass filter provides the approximate coefficients $A$. The filter output is given in (1) and (2) where $k$ varies from $-\infty$ to $\infty[22]$.

$$
\begin{aligned}
& Y_{\text {high }}=\sum x[k] h[2 n-k] \\
& Y_{\text {low }}=\sum x[k] g[2 n-k]
\end{aligned}
$$

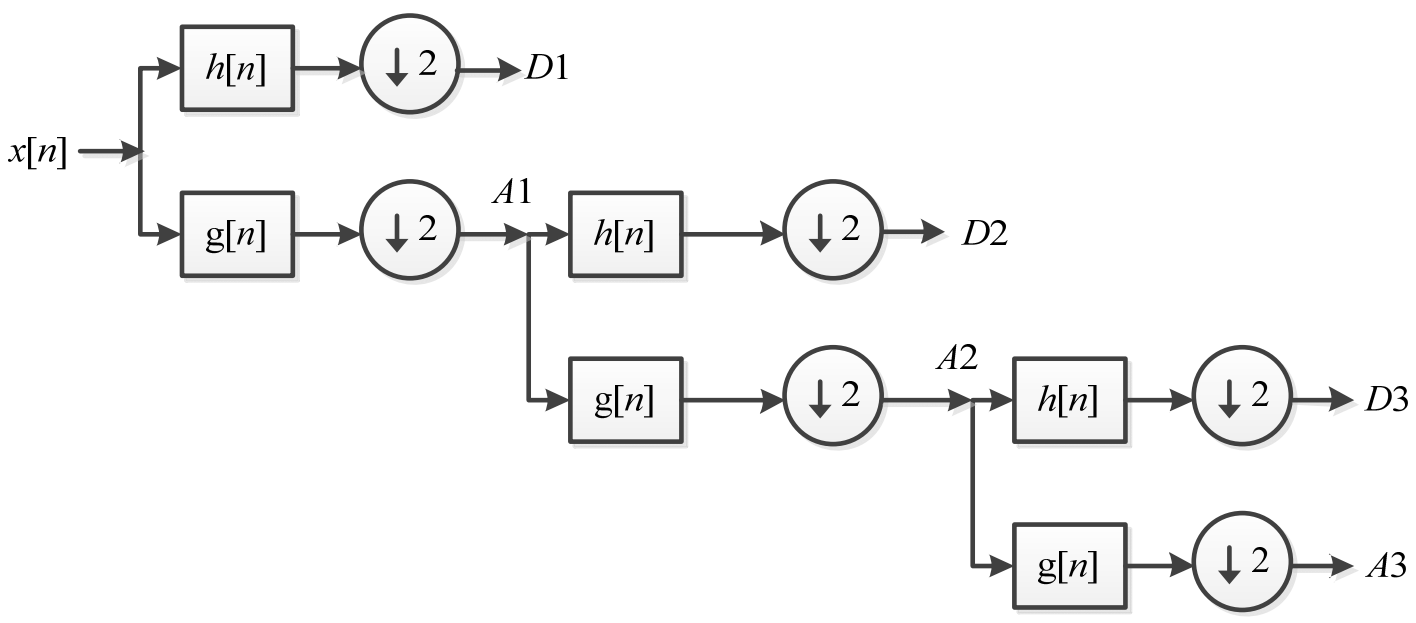

Figure 2. Wavelet decomposition tree up to level 3

We have decomposed the EEG signal in both time and frequency domain using DWT to select the EEG bands. The decomposed signals are then used to compute different features. We have used db4 wavelet which was found suitable after some trial and error attempts up to decomposition level four to six. Fourth, fifth and sixth level coefficients of wavelet decomposition corresponds the beta, alpha and theta band of frequencies respectively. We have used the beta band for features extraction.

There are numbers of time and frequency domain features used in literatures. Most of the cases magnitude, frequency, energy, power, and different statistical measures are considered for features calculation. After a rigorous study, we have considered eight effective features which are described below. Let, $N$ is the length of the EEG signal $x[n]$. 
i. Energy:

Energy of a signal can be defined as a simple square integral. It might be an important feature of EEG signal. For discrete EEG sequence the energy can be calculated as:

$$
\text { Energy }=\sum_{n=1}^{N}\left|x[n]^{2}\right|
$$

ii. Scale variance $(S V)$ :

Scale variance is measure of log-variance that can be expressed as:

$$
S V=\log (\operatorname{var}(x[n])) / \log 2
$$

iii. $R M S$ value:

The root mean square (RMS) is a statistical measure of varying signals which can show the strength of signal. The average power of a signal is square of its RMS value. The RMS value can be calculated as:

$$
R M S \text { value }=\sqrt{\frac{\sum_{n=1}^{N} x[n]^{2}}{N}}
$$

iv. Roll off:

The rolloff is a measure of the frequency below which $85 \%$ of the magnitude distribution of the spectrum is concentrated. It is also a measure of spectral shape and can be written as:

$$
R=0.85 \times \sum_{n=1}^{N / 2}|x[n]|
$$

v. Variance:

Variance is the mean value of the square of the deviation of the signal. However, mean of EEG signal is close to zero. Hence, we calculate the variance of EEG following the equation:

$$
V A R=\frac{1}{N-1} \sum_{n=1}^{N}\left|x[n]^{2}\right|
$$

vi. Approximate entropy (ApEn):

Approximate entropy is a measure of randomness or regularity [23]. A low value of the entropy indicates that the time series is deterministic; on the other hand, a high value indicates the randomness. ApEn has been used to characterize different biomedical events. For $\operatorname{ApEn}(m, r, N)$, two input parameters, a run length $m$, and a tolerance window $r$, must be considered for $N$ length time series. If $N$ data points form a time series $X=[x(1)$, $x(2), x(3), \ldots, x(N)]$., we can compute $A p E n$ as follows:

a) Form a subsequence of length $N-m+1$ consisting $x[1], x[2], \ldots ., x[N-m+1]$. Similarly, different subsequences are: $X[i]=[x(i), x(i+1), \ldots, x(i+m-1)]$, where $i=1,2, \ldots, n-m+1$

b) Calculate the distance $d[X(i), X(j)]$ between $X(i)$ and $X(j)$, as the maximum absolute difference between their respective scalar components:

$$
d[X(i), X(j)]=\max _{\mathrm{k}=1,2, . . \mathrm{m}}|x(i+k-1)-x(j+k-1)|
$$

c) For a given $X(i)$, count the number of $j,(j=1,2, \ldots, N-m+1)$ so that $d[X(i), X(j)] \leq r^{*} S T D$. Where, $S T D$ is the standard deviation of sequence and $r$ can be varying between 0 and 1 . The total counting number can be denoted as $\theta(i)$, where $i=1,2, . ., N-m+1$. Then, define a parameter $C_{r}^{m}(i)$ as below and compute it for each $i$.

$$
C_{r}^{m}(i)=\theta(i) /(N-m+1)
$$


d) Compute the natural logarithm of each $C_{r}^{m}(i)$ and average it over $i$,

$$
\varphi^{\mathrm{m}}(r)=\frac{1}{N-m+1} \sum_{i}^{N-m-1} \log _{e}\left(C_{r}^{m}(i)\right)
$$

e) Increase the dimension $m$ to $m+1$. Repeat steps a)-d) to get $C_{r}^{m+1}(i)$ and $\varphi^{m+1}(r)$.

f) ApEn can be calculated by the following formula:

$$
\operatorname{ApEn}(m, r, N)=\varphi^{m}(r)-\varphi^{m+1}(r)
$$

We have compute ApEn taking the value of $r$ as 0.15 . vii. Zero Crossing (ZC): calculated as:

Zero crossing indicates the number of times the EEG signal crosses the zero line. It can be

$$
\begin{aligned}
& Z C=\sum_{n=1}^{N-1} \operatorname{sgn}\left(x_{n}^{*} x_{n-1}\right) \cap\left|x_{n}-x_{n-1}\right| \geq \text { Threshold } \\
& \text { where, } \operatorname{sgn}(x)= \begin{cases}1, & x \geq \text { Threshold } \\
0, & \text { Otherwise }\end{cases}
\end{aligned}
$$

viii. Modified Mean Absolute Value (MMAV):

Mean Absolute Value (MAV) is the moving average of full-wave rectified EEG. It is calculated taking the average of absolute value of the EEG signal. $M M A V$ is the extension of $M A V$ with additional weighting function $w[n]$ as shown in equation (13).

$$
M M A V=\frac{1}{N} \sum_{n=1}^{N} w[n]|x[n]|
$$

where, $w[n]$ are taken as 1 for the samples $0.25 N \leq n \leq 0.75 N$ and 0.5 for rest of the samples.

\subsection{Classification using Neural Networks}

Artificial neural networks (ANNs) are similar to biological neuronal networks that are useful for pattern recognition, classification etc [20]. ANN learning is accomplished by training algorithms based on the learning mechanisms of biological neurons. There are various types of neural network varying fundamentally in the way they learn. In this study, a feed forward back propagation neural network and a probabilistic neural network are used.

A feed forward back propagation neural network consists of a number of simple neurons like processing unit. Every unit in a layer is connected with all the units in the previous layer. Along with input and output layers of neurons, hidden processing layer is also used to hold the nonlinearity and complexity of the problem. Selection of proper number of hidden layers is important. The proposed feed forward back propagation neural network structure is shown in Fig. 3. We have considered 8 abovementioned features. Therefore, neural network is designed with eight input nodes and one output node. The number of nodes in hidden layer has been set on trial-and-error basis. Sigmoid transfer function has been chosen to deal with nonlinearity. The network is trained with gradient descent algorithm. Performance of the network is specified as Mean Square Error (MSE). The training was stopped when the MSE between the network outputs and the targets was lesser than or equal to 0.00001 . The learning rate is fixed at 0.05 . The number of training epochs was fixed uniformly at 3500 . 


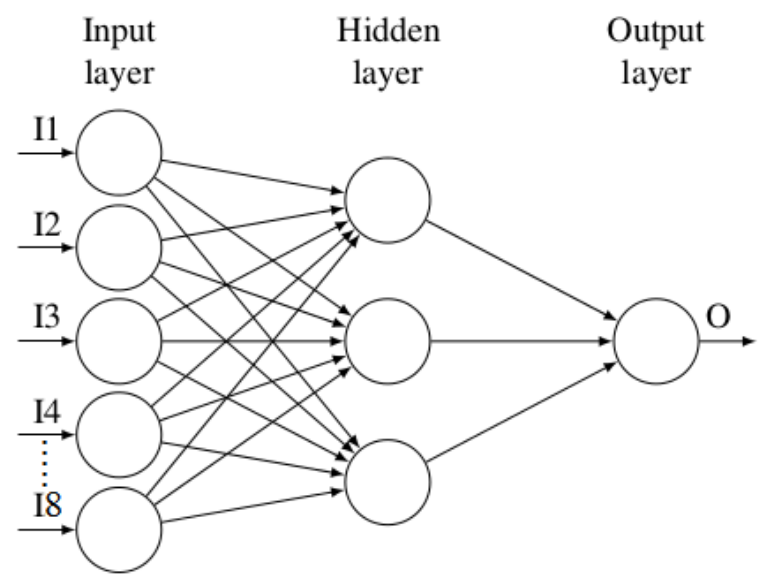

Figure 3. Proposed back propagation neural network structure

We have used a probabilistic neural network to find a better left and right hand movements signals classifier comparing to classical BP based NN. PNN has speedy training process with innately parallel structure and one-pass training effort [24]. It does not need an iterative training process and the training time is just the loading time of the training matrix. Therefore, learning rate of PNN is faster than many neural networks models, which increase its efficacy for real time applications. The input layer part distributes the input to the neurons in the pattern layer. Receiving the pattern from the input layer, the neuron of the pattern layer computes its output in accordance to the probability density function (pdf) for a single sample.

The PNN architecture has three layers: the input layer, the radial basis layer, and the output layer as shown in Fig. 4. The input vector $\mathbf{p}$ represents the feature vector with size $8 \times 1, Q$ is the number of input/target pair datasets and $K$ is the number of classes. In this algorithm, at the first layer input vectors calculate the distance from the training input and produces a new vector which is closed with the training input. Then, the radial basis layer sums all the contributions of the input vector and produces an output vector of probabilities. In radial basis layer, vector distances, $\|\mathbf{W}-\mathbf{p}\|$ of dimension $Q \times 1$ are calculated using dot product between input vector $\mathbf{p}$ and the each row of weight matrix $\mathbf{W}$ with dimension of $Q \times 8$. Then, the bias vector $\mathbf{b}$ is combined with $\| \mathbf{W}$ - $\mathbf{p} \|$ by an element-by-element multiplication. We have used radial basis transfer function as, $\operatorname{radbas}(\mathrm{n})=e^{-n^{2}}$. The spread value of the radial basis function was used as a smoothing factor which was considered as 0.1 . Then, the output of radial basis layer is found as: $a_{i}=$ $\operatorname{radbas}\left(\left\|\mathbf{W}_{i}-\mathbf{p}\right\| . \times \mathbf{b}_{\mathrm{i}}\right)$, where $\mathbf{W}_{i}$ is the ith row of $\mathbf{W}$, and $\mathbf{b}_{i}$ is the $i^{\text {th }}$ element of bias vector $\mathbf{b}$.

Finally, in the output competitive layer, the vector $\mathbf{a}$ is initially multiplied with layer weight matrix $\mathbf{M}$ to compute an output vector $\mathbf{d}$. There is no bias in this layer. The competitive function is used to pick the maximum probabilities and produces ' 1 ' for the intended class and ' 0 ' for the other class.

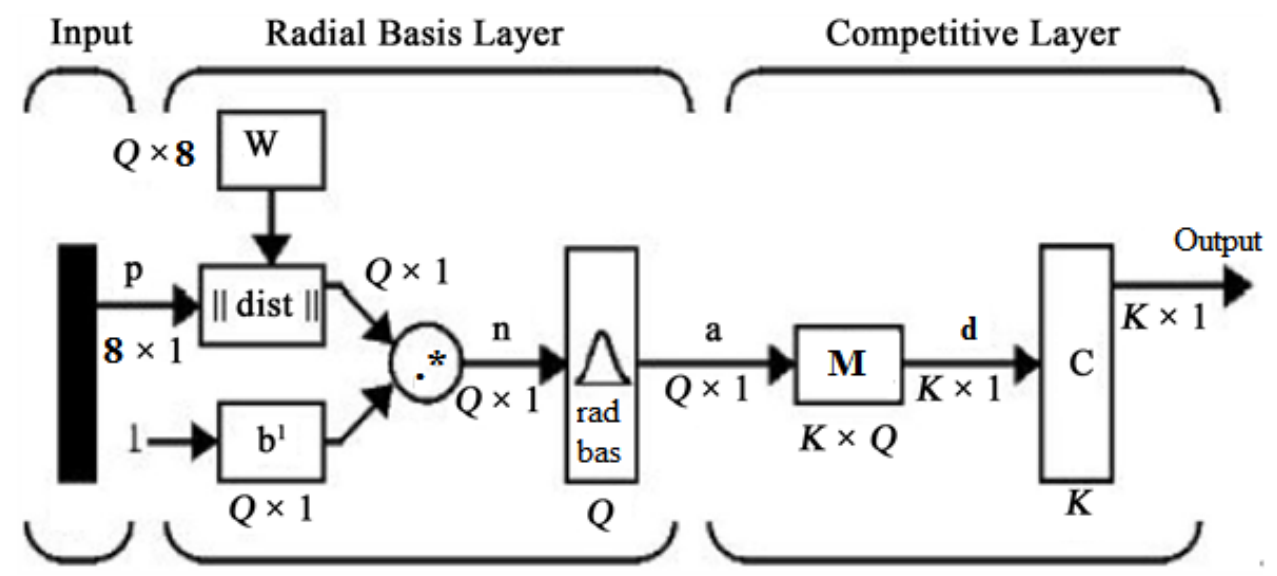

Figure 4. Probabilistic neural network structure 


\section{RESULT ANALYSIS}

We have used experimental EEG data of left and right hand movement. The dataset was collected from the website [25]. The subject of the dataset is a 21 year old, right handed male with no known medical conditions. The EEG consists of actual random movements of left and right hand recorded with eyes closed. There are nineteen electrodes. The order of the electrodes is FP1 FP2 F3 F4 C3 C4 P3 P4 O1 O2 F7 F8 T3 T4 T5 T6 FZ CZ PZ. The EEG signals was acuisited using Neurofax EEG System and power line frequency was $50 \mathrm{~Hz}$. The length of the signal for each electrode is 3200 samples and sample rate is $500 \mathrm{~Hz}$. Figure 5 shows the raw EEG data for left and right hand movement. The raw signals are then decomposed in different bands using discrete wavelet transform which are shown in Fig. 6. Selection of decomposition levels depends on alpha, beta and theta frequency band which was found in five, fourth and sixth decomposition respectively. Most of the computation and processing in this study are performed in MATLAB [26].

The performance of the classification of the EEG data is evaluated in terms of the three parameters i.e., sensitivity (SE), specificity (SP) and accuracy.

Sensitivity (SE) indicates the capacity of correctly identified positive cases and defined as:

$S E=\frac{T P}{T P+F N} ; \mathrm{TP}=$ True Positive and $\mathrm{FN}=$ False Negative.

Specifity (SP) indicates the capacity of correctly identified negative cases and expressed as:

$S P=\frac{T N}{F P+T N} ; T N=$ True Negative and $F P=$ False Positive

Accuracy indicates that the proportion of correct classified events. It can be calculated as:

Accuracy $=\frac{T P+T N}{T P+F P+T N+F N}$
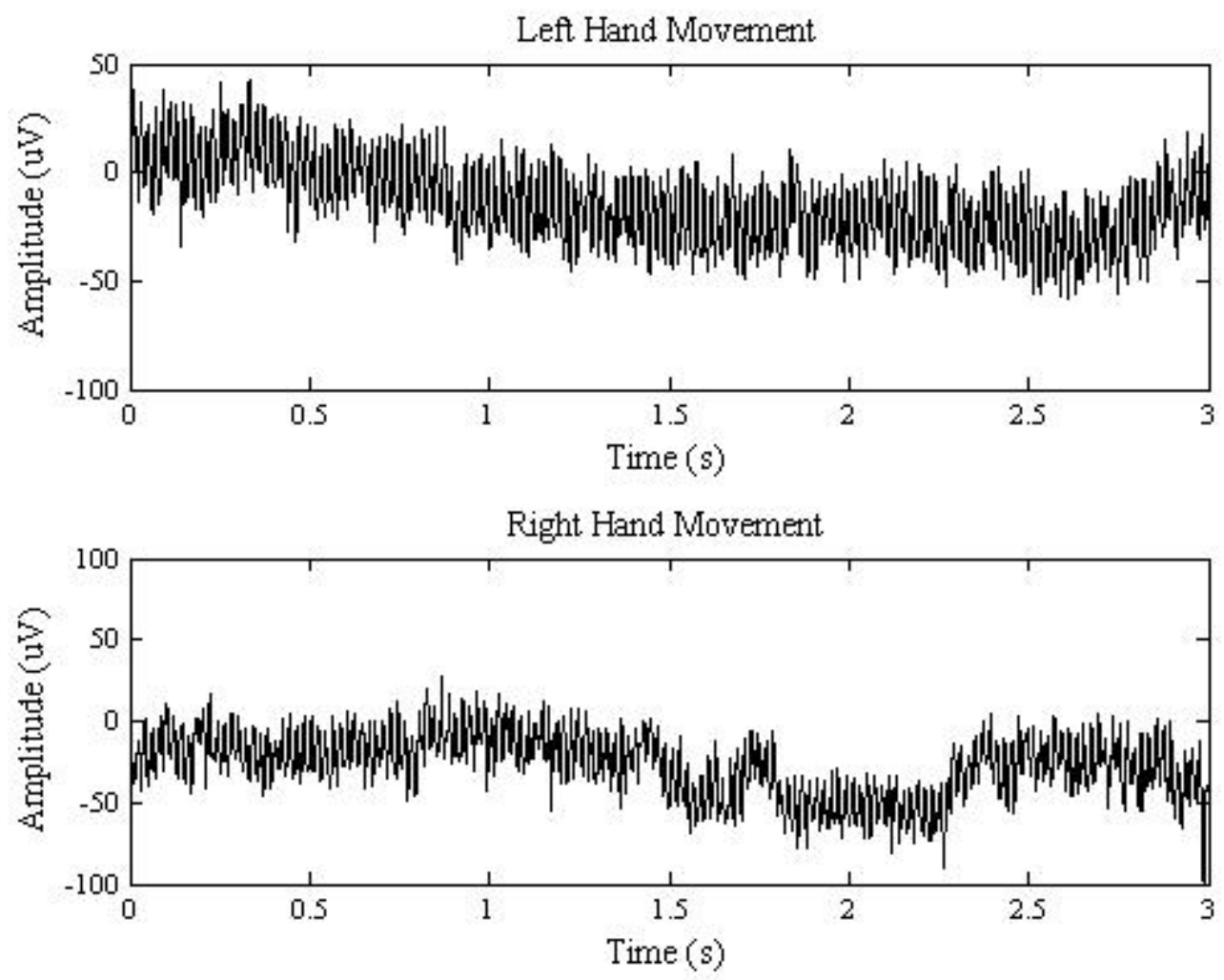

Figure 5. Raw EEG data for left and right hand movement 

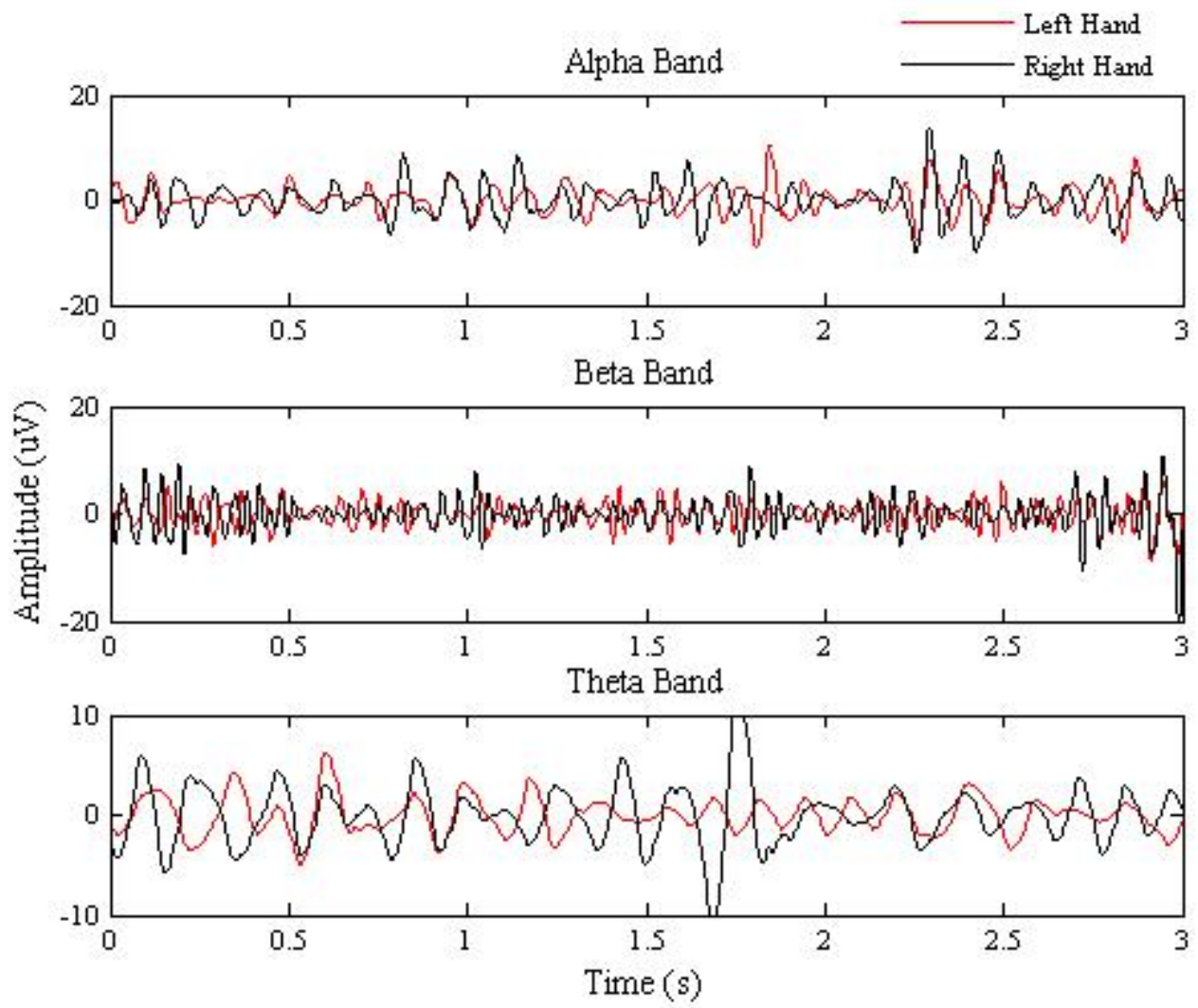

Figure 6. Separated alpha beta, and theta band from EEG using DWT

We have used $8 \times 57$ left hand datasets and $8 \times 57$ right hand datasets which were extracted from beta band for training and testing datasets. We have compared the performance of BP-NN and PNN classifier to find the better classifier. Table 1 and 2 show the result of two classifiers performance used in this study. Table 1 indicates the number of correct and false classifications of left and right hand movements EEG signal using BP based NN and PNN. Table 2 shows the comparison of overall performances by considering sensitivity, specificity and overall accuracy. The sensitivity of BP and PNN has been found as $86.35 \%$ and $91.74 \%$ respectively. The specificity of BP and PNN has been found as $98.31 \%$ and $100 \%$ respectively. The overall accuracy of BP and PNN is calculated as $88.9 \%$ and $99.1 \%$. From these data and performance parameter, it can be concluded that PNN has better classification rate than BP. Moreover, the quick training process with inherently parallel structure of PNN will inevitably speed up classifier decision execution time and improve its effectiveness fo real time applications.

Table 1. Classification Results of Hand Movement EEG Signals

\begin{tabular}{lcccc}
\hline Hand & \multicolumn{2}{c}{ BP } & \multicolumn{2}{c}{ PNN of Classification } \\
& No. of Classification & Correct & False \\
Left & Correct & False & 56 & 1 \\
Right & 48 & 9 & 57 & 0 \\
\hline
\end{tabular}

Table 2. Sensitivity, Specificity, and Accuracy of the Classifiers

\begin{tabular}{cccc} 
& Sensitivity & Specificity & Accuracy \\
\hline BP & $86.35 \%$ & $91.74 \%$ & $88.9 \%$ \\
PNN & $98.31 \%$ & $100 \%$ & $99.1 \%$ \\
\hline
\end{tabular}




\section{CONCLUSION}

The main focus of this study is to detect left and right hand EEG signal finding effective classifier. The EEG bands have been taken out through discrete wavelet transform. Eight extracted features from beta band have been used to train a classical back propagation based neural network and a probabilistic neural network in order to find effective classifier for left and right hand movement signals discrimination. Fifty seven left and right hand movement EEG features datasets were used in this study. The classification performance parameters show that PNN (99.1\%) has better classification rate than the BP (88.9\%). Also, the fast training process with innately parallel structure of PNN will inevitably speed up classification time and enhance its usefulness in real time applications. The findings of this study are expected to be useful in artificial hand movements through brain computer interfacing for bio-rehabilitation applications.

\section{ACKNOWLEDGEMENTS}

The authors would thank the authority of Khulna University of Engineering \& Technology, KUET, Bangladesh and the Brain Computer Interface research Lab at NUST, Pakistan for EEG data.

\section{REFERENCES}

[1] M.A. Lebedev and M.A.L. Nicolelis, "Brain-Machine Interfaces: Past, Present and Future", Trends in Neurosciences, vol. 29, pp.536-546, 2006.

[2] L.R. Hochberg, et al., "Neuronal Ensemble Control of Prosthetic Devices by a Human with Tetraplegia", Nature, vol. 442, pp. 164-171, 2006.

[3] J.R. Wolpaw, et al., "Brain-Computer Interface Technology: A Review of the First International Meeting", IEEE Transactions on Rehabilitation Engineering, vol. 8, pp.164-173, 2000.

[4] T. Ebrahimi, J.M. Vesin, and G.Garcia, "Brain-computer interface in Multimedia Communication", IEEE Signal Processing Magazine, vol. 20, pp. 14-24, 2003.

[5] S. Sanei and J. Chambers, EEG Signal Processing, John Wiley \& Sons, England, 2007.

[6] G. Pfurtscheller, C. Neuper, A. Schlögl, and K. Lugger, "Separability of EEG Signals Recorded During Right and Left Motor Imagery Using Adaptive Autoregressive Parameters", IEEE Transactions on Rehabilitation Engineering, vol. 6, pp. 316-325, 1998.

[7] E. Haselsteiner and G. Pfurtscheller, "Using Time-Dependent Neural Networks for EEG Classification", IEEE Transactions on Rehabilitation Engineering, vol. 8, pp. 457-463, 2000.

[8] D. Najumnissa and T.R. Rangaswamy, "Detection and Classification of Epileptic Seizures using Wavelet feature extraction and Adaptive Neuro-Fuzzy Inference System", International Journal of Computational Engineering Research, vol. 2, pp. 755-761, 2012.

[9] Y. Song, "A review of developments of EEG-based automatic medical support systems for epilepsy diagnosis and seizure detection", Journal of Biomedical Science and Engineering, vol. 4, pp. 788-796, 2011.

[10] M.H. Alomari, A. Samaha, and K. Al Kamha, "Automated Classification of L/R Hand Movement EEG Signals using Advanced Feature Extraction and Machine Learning”, International Journal of Advanced Computer Science and Applications, vol. 4, pp. 207-212, 2013.

[11] A. Schloegl, C. Neuper, and G. Pfurtscheller, "Subject Specific EEG Patterns During Motor Imaginary", in Conference of the IEEE Engineering in Medicine and Biology Society, 1997. IEMBS 1997. Nineteenth Annual International, 1997, pp. 1530-1532.

[12] E.J. Costa and E.F. Cabral Jr., "EEG-Based Discrimination Between Imagination of Left and Right Hand Movements Using Adaptive Gaussian Representation", Medical Engineering \& Physics, vol. 22, pp. 345-348, 2000.

[13] F. Wang, K. Kim, S. Wen, Y. Zhang, and C. Wu, "EEG Based Automatic Left-Right Hand Movement Classification", in Control and Decision Conference, 2012. CCDC 2012 .I , 2012, pp. 1469 - 1472.

[14] F. Quandt, et al., "Single Trial Discrimination of Individual Finger Movements on One Hand: A Combined MEG and EEG Study", NeuroImage, vol. 59, pp. 3316-3324, 2012.

[15] M.A. Riheen, M.W. Rahman, and A.B.M.A. Hossain, "Selection of Proper Frequency Band and Compatible Features for Left and Right Hand Movement from EEG Signal Analysis", Conference on Computer and Information Technology, 2013. ICCIT 2013, Sixteenth International, 2013.

[16] H. Olkkonen, Discrete Wavelet Transforms - Biomedical Applications, InTech, Rijeka, Croatia, 2011.

[17] A. Nait-Ali, Advanced Biosignal Processing, Springer, 2009.

[18] A. Subasi and E. Ercelebi, "Classification of EEG Signals Using Neural Network and Logistic Regression", Computer Methods and Programs in Biomedicine, pp.87-99, 2005.

[19] S.J. Husain and K.S. Rao, "Epileptic Seizures Classification from EEG Signals Using Neural Networks", in Conference on Information and Network Technology, 2012, ICINT 2012. International, 2012 pp.269-273.

[20] S. Haykin, Neural Networks-A Comprehensive Foundation, Prentice Hall, NJ, 1999.

[21] S.G. Mallat, "A theory for multiresolution signal decomposition: the wavelet representation", IEEE Transactions on Pattern Analysis and Machine Intelligence, vol. 11, pp. 674-692, 1989.

[22] A. Aldroubi and M. Unser, Wavelets in Medicine and Biology. CRC Press, USA, 1996. 
[23] H. Ocak, "Automatic detection of epileptic seizures in EEG using discrete wavelet transform and approximate entropy”, Expert Systems with Applications, vol. 36, pp. 2027-2036, 2009.

[24] L. Rutkowski, New Soft Computing Techniques for System Modelling, Pattern Classification and Image Processing, Springer, 2004

[25] Available in: http://sites.google.com/site/projectbci/

[26] The MathWorks Inc., Massachusetts, USA. Available in: http://www.mathworks.com/

\section{BIOGRAPHIES OF AUTHORS}
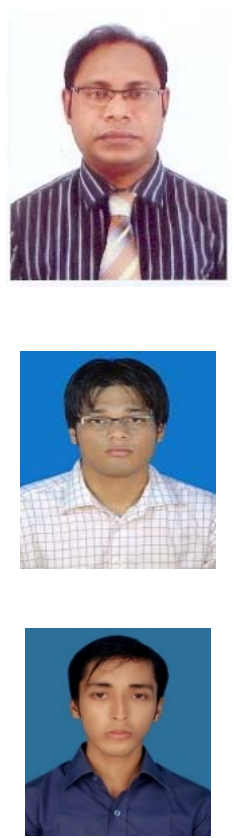

Dr. A.B.M. Aowlad Hossain received his B.Sc. in Electrical and Electronic Engineering (EEE) from Khulna University of Engineering \& Technology (KUET) and M.Sc. in EEE from Bangladesh University of Engineering \& Technology (BUET) in 2002 and 2005 respectively. He joined in KUET as lecturer in 2005. Dr. Hossain completed his Ph.D. in Biomedical Engineering from Kyung Hee University, Korea in 2012. Currently he is an Associate Professor in the Department of ECE, KUET. His research interests are biomedical signal and image processing, ultrasound imaging, and computer aided diagnisis etc. He is member of different professional societies and reviewers of different conferences and journals.

Mr. Md. Wasiur Rahman received his B.Sc. in Electronics and Communication Engineering (ECE) from Khulna University of Engineering \& Technology (KUET) in 2013. Currently he is preparing for higher studies and research. His research interests are biomedical signal and image processing.

Mr. Manjurul Ahsan Riheen received his B.Sc. in Electronics and Communication Engineering (ECE) from Khulna University of Engineering \& Technology (KUET) in 2013. Now he is working in cell phone research and development division of Walton Hi-Tech Industries Limited as an assistant engineer. His research interests are biomedical signal processing, signal processing for communications and sensor networks. 